\title{
Practical Guidance in the Conduct of a Scoping Review: Insights from Experience in the College of Public Health, University of the Philippines Manila
}

\author{
Carl Abelardo T. Antonio, MD, MPH, 1,2 Arianna Maever L. Amit, MAS, MS, 3,4 Ma. Sophia Graciela L. Reyes, BSPH, ${ }^{3,5}$ \\ Kim L. Cochon, MSPH, ${ }^{6}$ Jonathan P. Guevarra, MAN, ${ }^{7,8}$ Amiel Nazer C. Bermudez, MD, MPH,,, 10 \\ Chelseah Denise H. Torres, BSPH ${ }^{3,11}$ and Azar G. Agbon, BSN ${ }^{3}$ \\ ${ }^{1}$ Department of Health Policy and Administration, College of Public Health, University of the Philippines Manila, Manila, Philippines \\ ${ }^{2}$ Department of Applied Social Sciences, The Hong Kong Polytechnic University, Kowloon, Hong Kong \\ ${ }^{3}$ College of Public Health, University of the Philippines Manila, Manila, Philippines \\ ${ }^{4}$ School of Medicine and Public Health, Ateneo de Manila University, Pasig City, Philippines \\ ${ }^{5}$ Duke-NUS Medical School, Singapore \\ ${ }^{6} J C$ School of Public Health and Primary Care, Faculty of Medicine, The Chinese University of Hong Kong, Shatin, Hong Kong \\ 'Department of Health Promotion and Education, College of Public Health, University of the Philippines Manila, Manila, Philippines \\ ${ }^{8}$ College of Development Communication, University of the Philippines Los Baños, Los Baños, Laguna, Philippines \\ ${ }^{9}$ Department of Epidemiology and Biostatistics, College of Public Health, University of the Philippines Manila, Manila, Philippines \\ ${ }^{10}$ Department of Epidemiology, School of Public Health, Brown University, Providence, Rhode Island, United States of America \\ ${ }^{11}$ University of Santo Tomas Faculty of Medicine and Surgery, Manila, Philippines
}

\begin{abstract}
Objective. We aimed to provide practical guidance on the scoping review process, building on the methodologies and general steps outlined by Arksey and O'Malley, Levac et al. and The Joanna Briggs Institute.

Methods. We reviewed the methodologies of three scoping studies conducted by the authors in the College of Public Health, University of the Philippines Manila between 2016 and 2017. For each project, we outlined the steps, tools utilized, good practices performed, challenges encountered, and recommendations for improving the scoping review process in relation to existing guidelines. We compared the similarities and differences across the three reviews and guidelines to come up with a list of good practices and recommendations.
\end{abstract}

Results. We propose an expanded 10-step and iterative framework based on our analysis of three scoping studies: 1. Define your research question; 2 . Specify your research statement according to population, concept, and context; 3. Prepare the necessary tools, forms, and software packages; 4. Assemble your expert panel and/or consultants; 5. Develop your search strategy; 6. Implement the search strategy and retrieve identified studies; 7. Screen and assess studies for inclusion in the scoping review; 8. Chart the data; 9. Synthesize your results; and 10. Prepare your final report.

Conclusion. Scoping reviews as a method of evidence synthesis are increasingly gaining popularity among researchers due to the scope of what can be reviewed in a relatively short amount of time. With only three scoping studies informing our proposed methodology, other issues and challenges in the conduct of a scoping review may have been missed in the expanded framework presented in this paper. We suggest future studies to apply existing scoping review methodologies, to further enhance this rapidly evolving framework in research, policy, and practice.

Key Words: Scoping review, Review [Publication Type], Methods, Philippines, evidence synthesis

\section{INTRODUCTION}

Corresponding author: Carl Abelardo T. Antonio, MD, MPH Department of Health Policy and Administration College of Public Health University of the Philippines Manila 625 Pedro Gil Street, Ermita, Manila 1000, Philippines Email: ctantonio@up.edu.ph
Scoping reviews have been broadly defined as studies that rapidly map, synthesize, and analyze key concepts underpinning a research area; to identify gaps and the current state of understanding in the subject of interest; and/or assess the scope of research literature. ${ }^{1-4}$ Several methodological 
frameworks have been proposed for scoping reviews, three of which were considered in this current methodological note. ${ }^{2,5,6}$ These frameworks ${ }^{2,5,6}$ follow the same prescribed steps of: 1 . Identifying the research question; 2 . Identifying relevant studies; 3 . Selecting studies; 4 . Charting the data; 5. Collating, summarizing, and reporting the results; and 6. Conducting consultation exercises (Optional).

Given that scoping reviews are relatively new in health research and literature ${ }^{3}$ and have not been detailed in full extent ${ }^{5}$ even within the three existing frameworks, researchers are presented with the challenge of adapting their own strategies to conduct a scoping study (i.e., researchers develop their own techniques to operationalize the broad steps outlined in the existing frameworks, as was our experience). We aimed to provide practical guidance on the conduct of the scoping review process using insights from three scoping studies conducted by the authors in the College of Public Health, University of the Philippines Manila.

\section{METHODS}

We reviewed the methodologies of three scoping studies conducted by the authors in the College of Public Health, University of the Philippines Manila between 2016 and 2017..$^{7-9}$ Briefly, these scoping reviews followed the methodology described by The Joanna Briggs Institute (JBI), ${ }^{6}$ which in turn were informed by the work of Arksey and O'Malley ${ }^{2}$ and Levac et al., ${ }^{5}$ and aimed:

1. To synthesize evidence regarding the benefits, risks, costs, and barriers of paid maternity leave policies in the workplace; ${ }^{7}$

2. To support a landscape analysis to identify lessons, and describe existing models and frameworks of intersectoral collaborations in the context of dengue, malaria, and yellow fever; ${ }^{8}$ and

3. To address the question "What types of institutional and support systems were put in place by low- and middle-income countries in the implementation of compulsory service policies for selected health professionals?”. ${ }^{9}$

Each of these was broken down into steps according to the guidelines stipulated in the methodological framework for scoping reviews ${ }^{2}$ and the manual for JBI Scoping Reviews. ${ }^{6}$ For each project, we outlined the steps, tools utilized, good practices performed, challenges encountered, and recommendations for improving the scoping review process in relation to the three existing frameworks. ${ }^{2,5,6} \mathrm{We}$ compared the similarities and differences across the three reviews and guidelines to come up with a list of good practices and recommendations.

\section{RESULTS AND DISCUSSION}

\section{The Scoping Review Process: Good Practices and Recommendations}

We conducted three scoping studies between 2016 and $2017^{7-9}$ following the JBI Manual. ${ }^{6}$ We detailed our methodology according to the prescribed steps in a scoping review, ${ }^{6}$ and identified good practices and challenges in each of our studies. Based on our results and comparisons to three existing guidance documents on the scoping review process, $2,5,6$ we provided recommendations that would best address the current needs on scoping reviews.

We present our framework according to the following expanded steps:

1. Define your research question;

2. Specify your research statement according to population, concept, and context (PCC);

3. Prepare the necessary tools, forms, and software packages;

4. Assemble your expert panel and/or consultants;

5. Develop your search strategy;

6. Implement the search strategy and retrieve identified studies;

7. Screen and assess studies for inclusion in the scoping review;

8. Chart the data;

9. Synthesize your results; and

10. Prepare your final report.

\section{Define your research question}

The research question paves the way for an effective literature search and a clearly written report. Sometimes used interchangeably with 'research problem', there is general consensus that it has to be well-defined. ${ }^{10}$ The challenge is in determining which relevant questions may be studied through a scoping review and in rationalizing the need for an investigation into the topic of interest. ${ }^{11}$ This is addressed by various methods including observations, literature searches, interviews with patients and experts in the field, and an up-to-date understanding and awareness of the current context of the problem. ${ }^{11,12} \mathrm{We}$ emphasize that this is not an all-inclusive definition, such that the research question may be derived from a call for tenders or formulated with a collaborating or funding institution. In settings where the working group defines the research question themselves, it is important to identify existing studies, scoping reviews, and other synthesis papers that are relevant to the topic of interest. ${ }^{6,11}$ If such reviews exist, a justification must be included in the written report to discuss how the current review differs from others done on the same topic. ${ }^{6}$

In general, we recommend that the FINER criteria ${ }^{11,13}$ be used in the development of the research question as it highlights the attributes of a good question (Table 1). For this type of research, considerations must be given on the project timeline and breadth of scope of the databases, together with the sources of evidence that will be included. 
Table 1. FINER Criteria ${ }^{11,13}$

\begin{tabular}{ll}
\hline Feasible & Manageable in scope \\
\hline Interesting & $\begin{array}{l}\text { Getting the answer that intrigues investigators, } \\
\text { peers, and community }\end{array}$ \\
\hline Novel & Confirms, refutes, or extends previous findings \\
\hline Ethical & $\begin{array}{l}\text { Amenable to a study that the institutional } \\
\text { review board will approve }\end{array}$ \\
\hline Relevant & $\begin{array}{l}\text { To scientific knowledge, to clinical and health } \\
\text { policy, and to future research }\end{array}$ \\
\hline
\end{tabular}

As with any study, proposed scoping reviews must have a significant contribution to the field and topic of interest. We further highlight that ethical concerns may be of a lesser priority in these types of studies since scoping reviews only examine articles and documents without any involvement of human subjects. ${ }^{14}$

\section{Specify your research statement according to population, concept, and context (PCC)}

In any research undertaking, the question serves as a reference point throughout the study that guides protocol development, literature search, and implementation. ${ }^{15}$ For scoping reviews, both the research question and objectives should sufficiently address the three elements of PCC. ${ }^{6}$ This framework differs from that of other studies such as evidencebased medicine or clinical research that follows the $\mathrm{PICO}^{16}$ or PICOT criteria: ${ }^{12}$ population (patients); intervention (for intervention studies only); comparison group; outcome of interest; and time. Similarly, Cochrane reviews follow the PICO criteria, with the additional specification of the types of studies that will be included. ${ }^{17}$

Briefly, the PCC elements (Table 2) must be reflected consistently in the scoping review: in the title; research question; objectives; and inclusion criteria. ${ }^{6}$ These guide both the readers and reviewers in understanding the context of the study and making decisions about sources and references to include in the search. ${ }^{6}$ All three studies conducted by the authors followed this guideline. ${ }^{7-9}$

\section{Prepare the necessary tools, forms, and software packages}

Each step in the scoping review process requires different tools, forms, and software packages. We propose that these be prepared before the actual conduct of the three-step search strategy. ${ }^{6}$ The inclusion of this step early in the process will avoid delays in the search proper and increase efficiency by allotting enough time to develop data

Table 2. Population, Concept, and Context in a Scoping Review 6

\begin{tabular}{ll}
\hline Population & $\begin{array}{l}\text { Which types of participants (e.g., age group, } \\
\text { sex) are you interested in? }\end{array}$ \\
\hline Concept & $\begin{array}{l}\text { What specific interventions and/or outcomes } \\
\text { will be included in your scoping review? }\end{array}$ \\
\hline Context & $\begin{array}{l}\text { What settings (i.e., geographic, cultural) will } \\
\text { you consider? }\end{array}$ \\
\hline
\end{tabular}

extraction tools or purchase the products needed. Preparation of data extraction forms in advance was done by only one of three reviews conducted by the authors. ${ }^{7}$ However, we could not conclude on the relative efficiency of this method compared to preparation of forms at a later stage where these are needed since we did not implement both methods on any single scoping review. We also suggest that at this stage, researchers construct dummy tables and graphs to be used for presenting results. These data visualizations, along with the PRISMA Flow Diagram, ${ }^{18}$ may be filled out and completed simultaneously during the search and extraction of data from relevant literature.

\section{Assemble your expert panel and/or consultants}

There are two contrasting recommendations on the conduct of a consultation exercise in the scoping review process: the original framework by Arksey and O'Malley noted this is an optional element, ${ }^{2}$ while the later publication by Levac et al made consultation an essential component of the scoping study methodology..$^{5}$ All three reviews conducted by the authors ${ }^{7-9}$ included consultations with at least one content expert and a methods expert throughout the process of the review to discuss and resolve any issues encountered. One other study included solicitation of feedback from experts using a modified Delphi process. ${ }^{8}$

Regardless of the approach to consultation, we indicated this as the fourth stage in our proposed framework, and not the last, ${ }^{2,5,6}$ due to its importance in achieving the aims of the scoping review. Specifically, consultation can aid in the development of the research question (i.e., better alignment with the knowledge or policy gaps), refinement of the search strategy, identification of additional references or records, especially grey literature, and validation of findings., Experts can include those who are well-versed on the topic or research area, program or policy (e.g., practitioner, advocate), and/or approach (e.g., librarian, evidence synthesis specialist). We recommend that the expert panel and/or consultants be actively involved at each stage of the review to ensure that all insights and suggestions are incorporated in a timely manner.

\section{Develop your search strategy}

Once the research question and inclusion criteria for records or studies have been defined, additional eligibility criteria may be specified. These include restrictions to language, publication dates, and sources of evidence (e.g., peer-reviewed articles, grey literature). After these have been indicated adequately, a list of databases and other sources that will be utilized in the scoping review should be identified and selected. This step may be done with the help of an experienced librarian and/or information specialist. Depending on the time and available resources for the study, the following eligibility criteria may be included to be comprehensive in answering the central research question: ${ }^{2}$

1. More than the prescribed minimum of two databases; ${ }^{6}$

2. Languages other than English; 
3. A greater range of years; and

4. Grey literature.

Additional information that may also be specified in the search strategy include plans for hand-searching key journals and contacting authors for access to full-text articles and other necessary data. All three scoping reviews ${ }^{7-9}$ followed the first two steps of the JBI search strategy, ${ }^{6}$ while only one $^{7}$ conducted an additional search using the reference lists of the identified articles.

The three-step search strategy $y^{6}$ is as follows:

1. Initial limited search of at least two online databases of relevance to the research questions followed by an analysis of text words in the title, abstract, and index terms in the articles;

2. Second search utilizing all identified keywords and index terms across all databases and sources specified in the protocol; and

3. Third search using the reference lists or contact of authors for further information.

Any deviations from these steps must sufficiently be justified and included in the limitations of the study.

Two important aspects must be noted by researchers new to scoping reviews. First, the three-step process mentioned above is iterative in nature, with continual refinement applied to the search strategy as additional keywords or search terms are discovered through perusal of the literature. Second, it is desirable that search strategies be highly sensitive or inclusive (i.e., capture a high number of records initially) to avoid missing out on important studies or papers. An initial search with few hits may mean that the topic has not been fully researched yet, or that the search strategy has to be modified.

For all three reviews conducted, ${ }^{7-9}$ PubMed (MEDLINE) was identified as a primary database given that the topics were of biomedical nature (i.e., paid maternity leave, vector-borne diseases, return service agreement for health professionals). After refinement of the search strategy, we conducted the second search, ${ }^{7-9}$ as well as the third, ${ }^{7}$ in all identified relevant databases and providers that include PubMed, Scopus, PLoS, and ProQuest. We recommend documentation of the following at the minimum: date and time of search; number of records identified in a database; operators; and other techniques used in the search. This serves four main purposes:
1. For reproducibility of findings;

2. For ease in identification of any errors in the search conducted;

3. For documentation of any changes in this iterative process; and

4. For facilitation of report writing.

\section{Implement the search strategy and retrieve identified studies}

There is no general agreement at which levels the eligibility criteria should be applied. For instance, if one of the inclusion criteria is 'full-text article is available', exclusion of papers without full-text copies may be done prior to retrieval of these articles through other sources (e.g., authors themselves, digital repositories). In other cases where the timeline of the project accommodates such attempts, no filters or restrictions may be applied in all databases at the initial database search, and even during screening of article titles and abstracts. This decision is at the discretion of the researchers and expert panel, and justifications must be stated in the report. Additionally, it may be iterative such that criteria are applied throughout the search and screening process; this must be carefully documented as well. We provide some insights about the advantages and disadvantages of applying eligibility criteria at different stages of the scoping review process (Table 3).

Searching several databases and sources will result to some duplicate records. We merged and deduplicated the retrieved articles using different tools and software, namely EndNote, Microsoft Excel, and Covidence. Both EndNote and Microsoft Excel are sensitive to formatting variations and hence, may not be able to detect all duplicates. In such cases, manual deduplication may be done afterwards. If any are missed manually, these may be further deduplicated through an online platform developed for screening and evaluation of articles (e.g., Covidence). Deduplication can also be performed using other reference managers (e.g., Mendeley, Zotero), although the authors have not used these tools in any of the three scoping studies referenced in this paper.

\section{Screen and assess studies for inclusion in the scoping review}

Prior to screening and evaluation of studies, the population, concept, and context elements must be comprehensively understood by all researchers. This will avoid

Table 3. Advantages and Disadvantages of Applying Eligibility Criteria at Different Stages of the Scoping Review

\begin{tabular}{lll}
\multicolumn{1}{c}{ Step } & \multicolumn{1}{c}{ Advantages } & \multicolumn{1}{c}{ Disadvantages } \\
Initial search & - Manageable number of records & $\begin{array}{l}\text { May not be exhaustive and comprehensive to include all } \\
\text { relevant studies }\end{array}$ \\
\hline Second search & $\begin{array}{l}\text { - } \text { Mastest to conduct } \\
\text { number of databases and sources included }\end{array}$ & $\begin{array}{l}\text { May obtain enough relevant records to identify gaps in } \\
\text { knowledge and literature related to the topic }\end{array}$ \\
& - Relatively fast to conduct & \\
\hline Third search & - Exhaustive and comprehensive to include most \\
& studies of relevance & $\begin{array}{l}\text { - Not feasible where budget and time constraints are present } \\
\text { - Large volume of records to screen and assess }\end{array}$ \\
\hline
\end{tabular}


variations in the stringency of screening of relevant literature. For example, if 'malaria' is included as a criterion for Concept, the team should agree beforehand that articles mentioning malaria in passing but do not discuss the disease in detail, are not eligible for inclusion in the review. As emphasized by Levac et al.:

"This stage should be considered an iterative process of searching the literature, refining the search strategy, and reviewing articles for study inclusion [...] Reviewers should meet at the beginning, midpoint and final stages of the abstract review process to discuss challenges and uncertainties related to study selection and to go back and refine the search strategy, if needed".

These iterations were done by only one of the three reviews conducted. ${ }^{8}$ All retrieved articles were screened and assessed independently by at least two members of these projects: $:^{7-9} 1$. At the title and abstract level; and 2. At the full-text level.

At the screening of titles and abstracts, the assigned reviewers independently screened the articles for relevance to the study according to the pre-specified inclusion criteria. Conflicting evaluations were resolved either through consensus and/or third-party arbitration. All papers that met the inclusion criteria of the three scoping reviews were evaluated further through full-text assessments given that abstracts may be insufficient in describing the full extent of the article. ${ }^{2,19}$ Similar to screening of titles and abstracts, fulltext evaluations were conducted by at least two independent reviewers in these projects. ${ }^{7-9}$ Any conflicts were also resolved through consensus and/or a third-party arbitration. We suggest that all reasons for exclusion be described and documented at this stage, and indicated simultaneously in the PRISMA Flow Chart. ${ }^{18}$ Both screening and assessments may be done using Microsoft Excel, Covidence, or other tools accessible to the research team. The PRISMA Flow Chart is automatically produced when using Covidence for screening relevant literature.

\section{Chart the data}

'Charting' in a scoping review, akin to 'data extraction' in a systematic review, entails obtaining key information according to issues and/or themes related to the research question. ${ }^{5}$ Data extraction forms prepared in step 3 that contain at least the names of the authors, year of publication, study population, methodology, and outcome measures, ${ }^{2}$ will be filled out and continually updated (i.e., as additional records are retrieved from citations searching). Although the current studies only followed the JBI methodology, ${ }^{6}$ we agree with the recommendation provided by Levac and colleagues (2010) and reiterate in this paper: Charting the data should involve independent extraction from about five to ten studies using available data extraction forms (i.e., from other published studies; online resources such as Cochrane Learning Resources website) to determine consistency with the research question. Refinement of these forms should be done as needed to ensure alignment with the needs of the scoping study (e.g., inclusion or deletion of certain variables or data points).

\section{Synthesize your results}

This stage is said to be the most extensive in the scoping review process, and involves analysis, reporting, and interpretation of findings. ${ }^{5}$ Analysis, also referred to as collation and summarization, requires a descriptive numerical summary (e.g., number of records included, per year of publication, per source country, per PCC elements) and a thematic analysis., ${ }^{2,5}$ Accordingly, three components should be considered: ${ }^{5}$

1. Qualitative data analysis software to facilitate analyses of themes and content;

2. Best approach in relating the findings to readers (e.g., framework, tables); and

3. Implications situated in the context of research, policy, and practice.

In relation to these three elements, one review conducted by the authors utilized NVivo to generate a mind map of themes and coded data from the articles using the software. ${ }^{8}$ We suggest that reviewers be oriented on how to conduct data analysis (e.g., thematic analysis) and be reminded of the scope of the study prior to the conduct of this stage. These will ensure that only relevant data will be included, and incongruence in interpretation of data will be minimized. If codes are to be used, a codebook that defines these may be created to improve coding of text by multiple researchers. Themes and anecdotes must be properly cited. Meanwhile, the three reviews conducted by the authors applied several approaches in presenting the findings (i.e., framework, tables, graphs), given that various research questions are best represented in different ways. Lastly, our conclusions incorporated research and policy implications, and provided recommendations to stakeholders.

\section{Prepare your final report}

Depending on how the research question was formulated (i.e., by the research team, derived from a call for tenders, or with a collaborating or funding agency), the format of the report may follow the guidelines of the collaborating or funding institution, or the target journal. Generally, the final report includes the title of the scoping review, review authors, background and rationale, objectives, inclusion criteria, types of sources, search strategy, charting table or forms, results, discussion, conclusions, implications, and references. ${ }^{6}$

\section{CONCLUSIONS}

Scoping reviews are increasingly gaining popularity among researchers as a method of evidence synthesis due to the breadth and scope these can encompass in a relatively 
short amount of time. ${ }^{2,5} \mathrm{We}$ presented an expanded and iterative framework based on three scoping studies, ${ }^{7-9}$ building on previous methodologies:2,5,6

1. Define your research question;

2. Specify your research statement according to population, concept, and context;

3. Prepare the necessary tools, forms, and software packages;

4. Assemble your expert panel and/or consultants;

5. Develop your search strategy;

6. Implement the search strategy and retrieve identified studies;

7. Screen and assess studies for inclusion in the scoping review;

8. Chart the data;

9. Synthesize your results; and

10. Prepare your final report.

Even though only three scoping studies informed our proposed framework that may have been insufficient to cover other issues and challenges, thus limiting our list of recommendations, these were done based on different approaches for arriving at a research question; inclusion criteria; tools, forms, and software products utilized; expert panels and consultants involved; and search strategies developed. Consequently, these variations may have compensated for the limited number of studies included, ${ }^{7-9}$ and guidelines compared and contrasted. ${ }^{2,5,6} \mathrm{We}$ recommend future studies to apply existing scoping review methodologies, and further enhance this rapidly evolving framework for uptake in research, policy, and practice.

\section{Statement of Authorship}

All authors participated in data collection and analysis, and approved the final version submitted.

\section{Author Disclosure}

All authors declared no conflicts of interest.

\section{Funding Source}

No funding support.

\section{REFERENCES}

1. Anderson S, Allen P, Peckham S, Goodwin N. Asking the right questions: scoping studies in the commissioning of research on the organisation and delivery of health services. Health Res Policy Syst. 2008 Jul 9;6:7. doi: 10.1186/1478-4505-6-7.
2. Arksey H, O'Malley L. Scoping studies: towards a methodological framework. Int J Soc Res Methodol. 2005;8(1):19-32. doi:10.1080/ 1364557032000119616.

3. Davis K, Drey N, Gould D. What are scoping studies? A review of the nursing literature. Int J Nurs Stud. 2009 Oct;46(10):1386-400. doi: 10.1016/j.ijnurstu.2009.02.010.

4. Grant MJ, Booth A. A typology of reviews: an analysis of 14 review types and associated methodologies. Health Info Libr J. 2009 Jun;26(2):91-108. doi: 10.1111/j.1471-1842.2009.00848.x.

5. Levac D, Colquhoun H, O'Brien KK. Scoping studies: advancing the methodology. Implement Sci. 2010 Sep 20;5:69. doi: 10.1186/ 1748-5908-5-69.

6. The Joanna Briggs Institute. (2015) Joanna Briggs Institute Reviewers' Manual: 2015 edition. Adelaide: The Joanna Briggs Institute; 2002.

7. Amit AM, Asence A, Jurado JE, Antonio CA. Prioritizing maternal and child health through paid maternity leave policies in the workplace: A scoping review. Poster presented at: Fifth Global Symposium on Health Systems Research; 8-12 Oct 2018; ACC Liverpool, Liverpool, United Kingdom.

8. Reyes ML, Torres CH, Bermudez AC, Cochon KL, Roxas EA, Liao SP, Ortega DN, Silang AC, Uezono DR, Salamat MS, Antonio CT. Intersectoral collaborations for the prevention and control of vector borne diseases: a scoping review. Acta Med Philipp. 2019;53(4): 343-53.

9. Antonio CT, Guevarra JP, Medina PN, Avelino MD, Agbon AG, Sepe DC, Bardelosa DD, Cengca MM, Ting ML, Lara AB. Components of compulsory service program for health professionals in lowand middle-income countries: A scoping review. Perspect Public Health. 2020;140(1):54-61. doi: 10.1177/1757913919839432.

10. Supino PG, Borer JS. Principles of research methodology: a guide for clinical investigators. New York: Springer; 2012.

11. Farrugia P, Petrisor BA, Farrokhyar F, Bhandari M. Research questions, hypotheses and objectives. Can J Surg. 2010 Aug;53(4): 278-81.

12. Haynes RB. Forming research questions. J Clin Epidemiol. 2006 Sep;59(9):881-886. DOI:10.1016/j.jclinepi.2006.06.006.

13. Hulley S, Cummings S, Browner W. Designing clinical research, 3rd ed: Philadelphia: Lippincott Williams and Wilkins; 2007.

14. World Health Organization. Ethical standards and procedures for research with human beings [Online]. 2011 [cited 2018 Jul 02]. Available from: http://www.who.int/ethics/research/en/.

15. Thoma A, McKnight L, McKay P, Haines T. Forming the research question. Clin Plast Surg. 2008 Apr;35(2):189-93. doi: 10.1016/ j.cps.2007.10.009.

16. Sackett D, Straus S, Richardson WS, Rosenberg W, Haynes RB. Evidence-based medicine: How to practice and teach EBM. London: Churchill Livingstone; 1999.

17. O'Connor D, Green S, Higgins J. Chapter 5: Defining the review question and developing criteria for including studies. In: Higgins J, Green S, eds. Cochrane handbook for systematic reviews of interventions. Chichester: Wiley-Blackwell; 2008. pp. 83-94.

18. Moher D, Liberati A, Tetzlaff J, Altman DG; PRISMA Group. Preferred reporting items for systematic reviews and meta-analyses: the PRISMA statement. PLoS Med. 2009 Jul 21;6(7):e1000097. doi: 10.1371/journal.pmed.1000097.

19. Badger D, Nursten J, Williams P, Woodward M. Should all literature reviews be systematic? Evaluation and Research in Education. 2000;14(3 \& 4):220-230. doi:10.1080/09500790008666974. 